\title{
Biocolloid retention in partially saturated soils
}

\author{
Tammo S. Steenhuis ${ }^{1}$, Annette Dathe ${ }^{1}$, Yuniati Zevi ${ }^{1}$, Jennifer L. Smith ${ }^{1}$, Bin GaO ${ }^{1}$, \\ Stephen B. Shaw ${ }^{1}$, Dilkushi DeAlwis ${ }^{1}$, Samary Amaro-Garcia ${ }^{1}$, Rosemarie Fenrman ${ }^{1}$, \\ M. Ekrem CakmaK ${ }^{1}$, Ian C. Toevs ${ }^{1}$, Benjamin M. LiU ${ }^{1}$, Steven M. Beyer ${ }^{1}$, John T. CRIST ${ }^{1}$, \\ Anthony G. Hay ${ }^{1}$, Brian K. Richards ${ }^{1}$, David DiCarlo ${ }^{2}$ \& John F. McCarthy ${ }^{3}$ \\ ${ }^{1}$ Cornell University, Department of Biological $\&$ Environmental Engineering, Riley-Robb Hall, Ithaca, NY 14853, USA; \\ e-mail: tss1@cornell.edu \\ ${ }^{2}$ USDA/ARS National Sedimentation Laboratory, Oxford, MS 38655, USA \\ ${ }^{3}$ University of Tennessee, Knoxville, TN 37996, USA
}

\begin{abstract}
Unsaturated soils are considered excellent filters for preventing the transport of pathogenic biocolloids to groundwater, but little is known about the actual mechanisms of biocolloid retention. To obtain a better understanding of these processes, a number of visualization experiments were performed and analyzed.
\end{abstract}

Key words: colloid transport, colloid retention, microbial transport, microbial retention, partially-saturated soil, unsaturated porous media, pathogens

\section{Introduction}

Pathogenic microbes including bacteria, viruses and protozoa have been implicated in waterborne disease outbreaks (BARWICK et al., 2000; MACLER \& MERKLE, 2000). Almost all studies of pathogen mobility in soils have focused on the transport and retention of bacteria in saturated soils. However, pathogens generally enter the soil environment from land-applied wastes or septic systems at or near the soil surface, where the soil is typically unsaturated (i.e. only partially saturated with water). Unsaturated systems are much more complex and more poorly understood than water-saturated groundwater systems with respect to contaminant flow and retention (MCCARTHY $\&$ MCKAY, 2004). In addition to the retention that occurs in saturated soil, bacteria may interact with two additional classes of interfaces - air-water-solid (AWS) and air-water (AW) - in unsaturated soils. The area of these interfaces is highly variable depending on changes in soil moisture, and is thus highly impacted by transient wetting and drainage events such as storms or snowmelt.

\section{Methods}

To characterize transport, retention and remobilization of biocolloids in unsaturated soil, we used small flow cells packed with translucent silica sand (cleaned by combustion and rinsing) imaged using a confocal scanning laser microscope. A syringe inlet pump and peristaltic outlet pump control the chamber moisture content and flow rate. Fluorescent synthetic microsphere surrogates with sizes and properties similar to either pathogenic bacteria or Cryptosporidium parvum oocysts and varying degrees of hydrophily were injected into the flow cell as dilute suspensions. Bright field microscopy was used for detection of dyed blue or red colloids in backlighted chambers (Fig. 1). Fluorescent colloids are detected with a Leica TCS SP2 confocal scanning laser microscope (10x $0.40 \mathrm{UV}$ objective) which simultaneously records three different spectral channels: 1) fluorescent microsphere emissions (500 to $540 \mathrm{~nm}$ ) excited at $488 \mathrm{~nm}$ by an argon laser; 2) the water phase emissions ( 555 to $650 \mathrm{~nm}$ ) due to Rhodamine B stain excited at $543 \mathrm{~nm}$ by a green HeNe laser; and 3) transmitted visible light to show the location of the sand grains.

Imaging with confocal laser and bright field microscopes indicated that hydrophilic colloid retention occurred primarily at the air/water meniscus/solid $\left(\mathrm{AW}_{\mathrm{m}} \mathrm{S}\right)$ interface, as we termed it in ZEVI et al. (2005), denoting the region where between-grain water menisci diminish to a thin water film on the grain surface in connected pores where most of the water flows (Fig. 1a). Conversely, isolated unconnected pendular rings did not contain microspheres, as also can be seen in Fig. 1a. ZEVI et al. (2005) showed that microspheres were retained at the $\mathrm{AW}_{\mathrm{m}} \mathrm{S}$ interface where the water film thickness approximately equaled the microsphere di- 


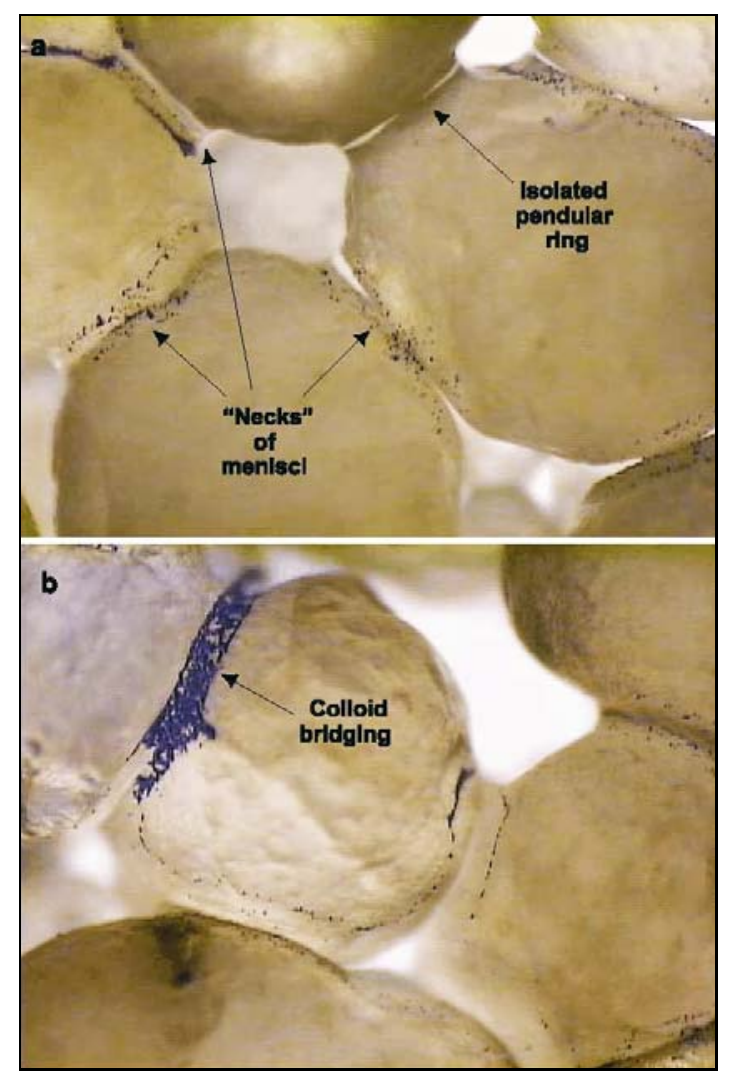

Fig. 1. Bright field images of colloid attachment: a) menisci (pendular water rings) between sand grains. Blue microspheres are visible in pores and interfaces participating in flow, while isolated menisci have no microspheres; b) Coagulated hydrophilic colloids form "bridges" between sand grains.

ameter. The greater retention efficiency for hydrophilic microspheres at this interface (where a greater portion of the microspheres were retained compared to the remainder of the solid/water interface) can be explained by the additional surface tension capillary potentials exerted on microspheres protruding from the water film at the interface, as discussed below. We also observed that hydrophilic microspheres readily attached to other microspheres already present at the $\mathrm{AW}_{\mathrm{m}} \mathrm{S}$ interface, as can be seen in the "bridge" of colloids starting at the $\mathrm{AW}_{\mathrm{m}} \mathrm{S}$ interfaces (Fig. 1b) observed by CRIST et al. (2005). In addition, the experiments of ZEVI et al. (2005) found that the mechanisms for more hydrophobic microsphere retention differed slightly. Microsphere distribution in flowing water played an important role in determining contact efficiency, with many more of these microspheres found near the water-solid (WS) interface. These microspheres were retained not only at the $\mathrm{AW}_{\mathrm{m}} \mathrm{S}$ interface but also at WS and AW interfaces, as their relative hydrophobicity impelled the microspheres to avoid water. The greater contact efficiency of these microspheres explains their greater retention observed in the literature. Another major factor controlling retention efficiencies of microspheres with low hydrophobicity was physical imperfections (surface

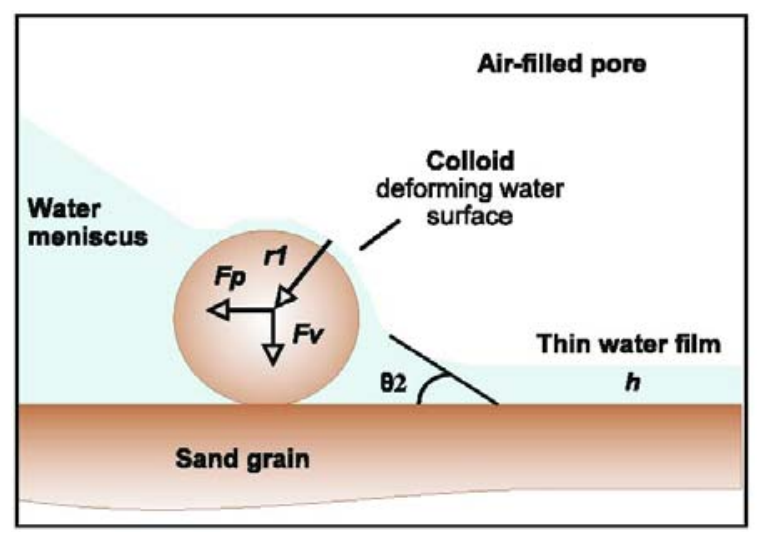

Fig. 2. Schematic of capillary forces $\left(F_{\mathrm{p}}\right.$ and $\left.F_{\mathrm{v}}\right)$ on colloid trapped at an $\mathrm{AW}_{\mathrm{m}} \mathrm{S}$ interface.

roughness and irregularities) of the sand grains. While some $0.8 \mu \mathrm{m}$ microspheres were observed being retained in thin water films, film straining played no significant role in the retention of larger microspheres.

A question that needs to be answered is why these colloids attach at $\mathrm{AWmS}$ interface. In the literature, DVLO forces have been always considered to be the main factor controlling the retention of colloids at the surfaces (either grain or water meniscus). DVLO forces, named after Derjaguin-Landau-Verwey-Overbeek, are a result of van der Waals, and double layer potential energies. These forces are typically effective over distances of less than $100 \mathrm{~nm}$. Because theory and visualizations both indicate that the hydrophilic colloids used in these experiments did not attach to either the grain (water/solid) or meniscus (air/water), it is unlikely that DVLO forces would dominate colloid retention at the intersection of both surfaces. As the first step in understanding colloid retention at the $\mathrm{AW}_{\mathrm{m}} \mathrm{S}$ interface, let us examine what is special about this interface.

As shown in Fig. 2, the water meniscus thins at the $\mathrm{AW}_{\mathrm{m}} \mathrm{S}$ interface from the bulk fluid to a much thinner film around the grain. Because a colloid (with a diameter less than $10 \mu \mathrm{m}$ ) is much smaller than the grain and the meniscus, for simplicity the grain surface, meniscus, and water film can all be assumed to be flat. The water film on the grain surface typically has a thickness in the submicron range and thus at some point a thickness similar to the colloid diameter. One can think of the meniscus as an elastic membrane that thermodynamically strives to have the smallest area. It will thus push back against any object that tries to protrude through the membrane, a property that is important for colloid retention. When colloids move in the liquid close to this interface, any irregularity in flow or the grain surface can cause the colloid to become impinged in a place where the flowpath becomes thinner than the colloid dimensions. Theoretically GAO et al. (2006) showed that a colloid near the $\mathrm{AW}_{\mathrm{m}} \mathrm{S}$ interface can be forced by its own momentum into a film that is thinner than its own diameter pushing the film outward. As a result, this de- 
formation will generate a capillary force perpendicular to the intersection points with the grain and the meniscus $(F)$. As shown in Fig. 2, this capillary force can be decomposed in two forces: a lateral force $\left(F_{\mathrm{p}}\right)$ which pushes the colloid back toward the bulk water, and a vertical force $\left(F_{\mathrm{v}}\right)$ which pins the colloid on the grain surface. Given the contact angle between water and the colloid $(\theta)$, the vertical capillary forces of colloid at the AWS interface can be expressed as:

$$
F_{\mathrm{p}}=2 \pi r_{1} \sigma \cos \left(\theta+\cos ^{-1} \frac{r_{1}}{r}\right) \cos \theta_{2}
$$

and

$$
F_{\mathrm{v}}=2 \pi r_{1} \sigma \cos \left(\theta+\cos ^{-1} \frac{r_{1}}{r}\right) \sin \theta_{2}
$$

where $\sigma$ is the liquid-vapor interfacial tension for water $\left(72.9 \mathrm{mN} \mathrm{m}^{-1}\right.$ at $\left.20^{\circ} \mathrm{C}\right), \theta_{2}$ is the water-grain contact angle, and $r_{1}$ is the radius of the contact area between colloid and the meniscus, which depends on the position of the colloid at the $\mathrm{AW}_{\mathrm{m}} \mathrm{S}$ interface.

The lateral force $\left(F_{\mathrm{p}}\right)$ tends to push the colloid away from the $\mathrm{AW}_{\mathrm{m}} \mathrm{S}$ interface into the bulk solution (as one might expect from a thermodynamic view to make the meniscus smaller and as demonstrated for a suspended film by SUR \& PAK, 2001). Our visualizations indicate, however, that colloidal particles remain at the $\mathrm{AW}_{\mathrm{m}} \mathrm{S}$ interfaces. This is because the vertical force $\left(F_{\mathrm{v}}\right)$ perpendicular to the grain pins the colloid



Fig. 3. Blue microspheres left in rings by evaporating water drop.

at the grain surface which may generate a static friction force $\left(F_{\mathrm{r}}\right)$ to balance the lateral force $\left(F_{\mathrm{p}}\right)$. If $F_{\mathrm{r}}$ is big enough, which depends on the magnitude of $F_{\mathrm{v}}$ and the surface roughness (static friction coefficient), to overcome the lateral force, it will prevent the colloid moving back into flow. Thus the colloids are "caught" at the $\mathrm{AW}_{\mathrm{m}} \mathrm{S}$ and will only be released when the interface moves or disappears (as shown by KELLER et al. in their experiments, although their theoretical explaination was slightly different). This indicates that both capillary force and surface roughness are very important to colloid retention and release in unsaturated porous media.

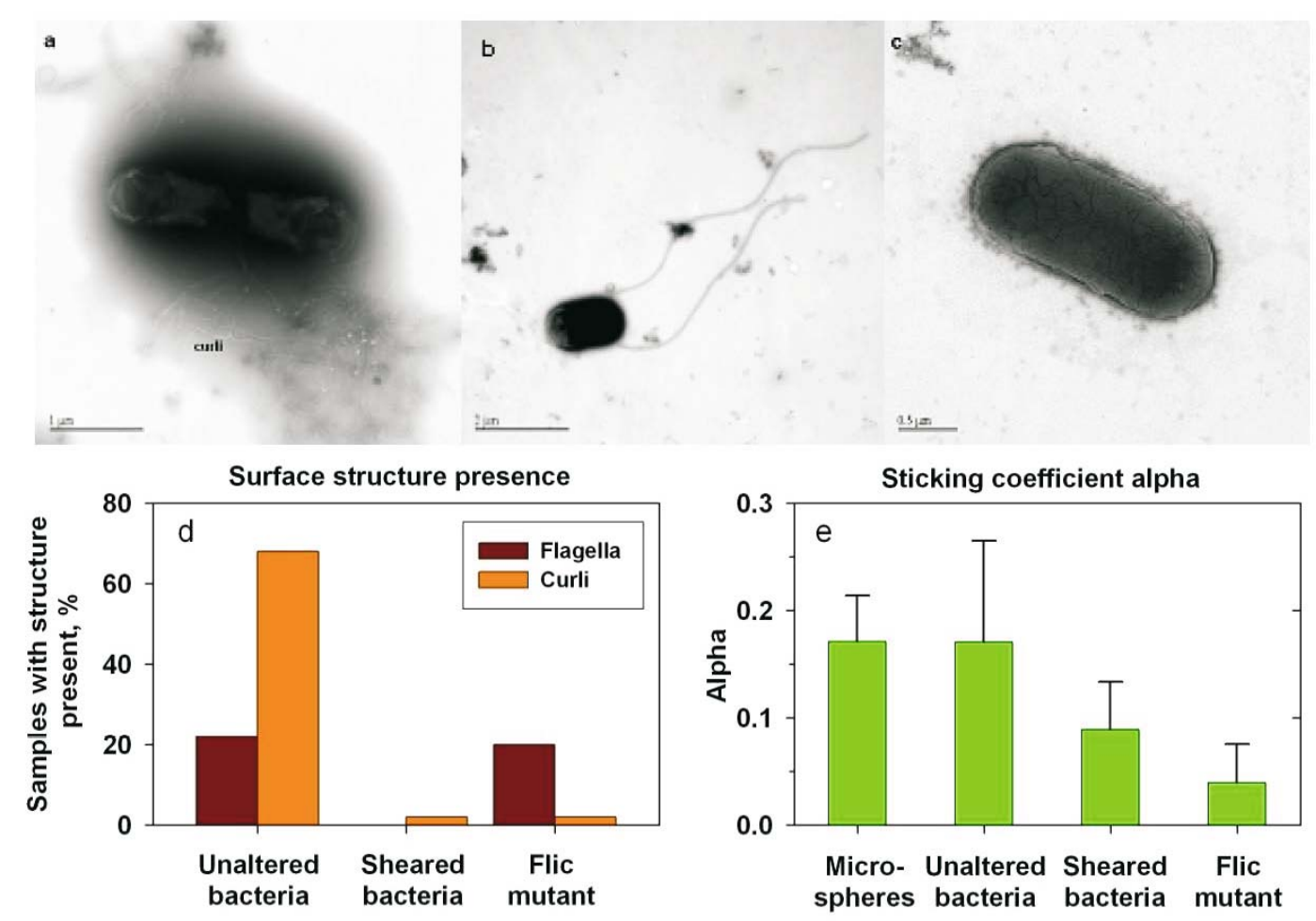

Fig. 4. Bacterial surface structures and transport: transmission electron microscope images of $E$. coli PHL 628 GFP-1 bacteria made with negative staining: a) untreated, with thin curli visible above and below bacteria; b) sheared via vortexing to remove curli, with flagella still visible; and c) flic mutant that expresses neither curli nor flagella; d) occurrence of surface structures in different treatments; and e) resulting transport sticking coefficient (alpha) determined in flow chamber tests. 
The attachment mechanisms occurring at the $\mathrm{AW}_{\mathrm{m}} \mathrm{S}$ interface are not limited to unsaturated porous media but can be seen in evaporating water drops as well. An example is shown in Fig. 3 where blue microspheres were pinned in successive rings at the $\mathrm{AW}_{\mathrm{m}} \mathrm{S}$ interface at the edge of a water droplet as it evaporated. The evaporation causes the meniscus of the drying drop to "stretch" in time more over the meniscus imposing a larger and larger capillary force. In order for a meniscus to move from a pinned location the lateral force component of the capillary force $\left(F_{\mathrm{p}}\right)$, need to exceed the friction force, $\left(F_{\mathrm{r}}\right)$ on the colloids. This friction force is dependent on the vertical capillary force, $\left(F_{\mathrm{v}}\right)$ and the static friction coefficient. Once the meniscus moves, the dynamic friction force which is less than the static friction will try to stop the colloids. As the colloids move $\mathrm{n}$ at one point $F_{\mathrm{p}}$ and $F_{\mathrm{v}}$ balance to stop the movement. The meniscus will not move unless the lateral force can exceed the friction force as explained above.

We used the same experimental apparatus as described above and added a range of green fluorescent protein (gfp)-expressing E. coli PHL 628 GFP-1 bacteria to test whether our visualization techniques could be applied to the retention of bacteria. Not only was this successful, but an initial set of experiments went on to determine the effects of various exocellular bacterial surface structures (flagella and curli) on the bacterial sticking coefficient (alpha) in the flow chamber. Unaltered bacteria (Fig. 4a) were compared to those with fewer structures that encourage attachment: a sheared bacteria that experienced the loss of curli via vortexing (Fig. 4b), and a flic mutant that had neither flagella nor curli (Fig. 4c). Observations in Fig. 4d show that the extent of exocellular structures differed substantially as a result of these treatments. Although additional tests will be needed to confirm findings, the trend in Fig. 4e indicated that while unaltered bacteria had retention characteristics similar to the microsphere surrogates, the altered bacteria were less likely to be retained.

Implicit in Fig. 4 is the fact that our confocal microscope configuration makes it possible to quantify fluorescent microsphere and biocolloid retention. To do so, a sequence of confocal microscope images acquired from the same location is saved as a stack file. Fig. 5a shows a reconstructed confocal laser microscope image of fluorescent colloids (yellow fringes on sand grains), dyed water (the reddish pendular ring between grains) and the sand grains. Image analysis techniques are used to transform the images to black and white (binary) images by thresholding the argon laser channel images (in which the microsphere location information is stored). This discriminates pixels as representing either particles or background. No further image enhancement is needed except where interference may be encountered from spurious light reflecting from the water surface. The interfacial regions in which the counting will take place are delineated such as is shown in Fig. 5b. The total number of black pixels in the selected regions are

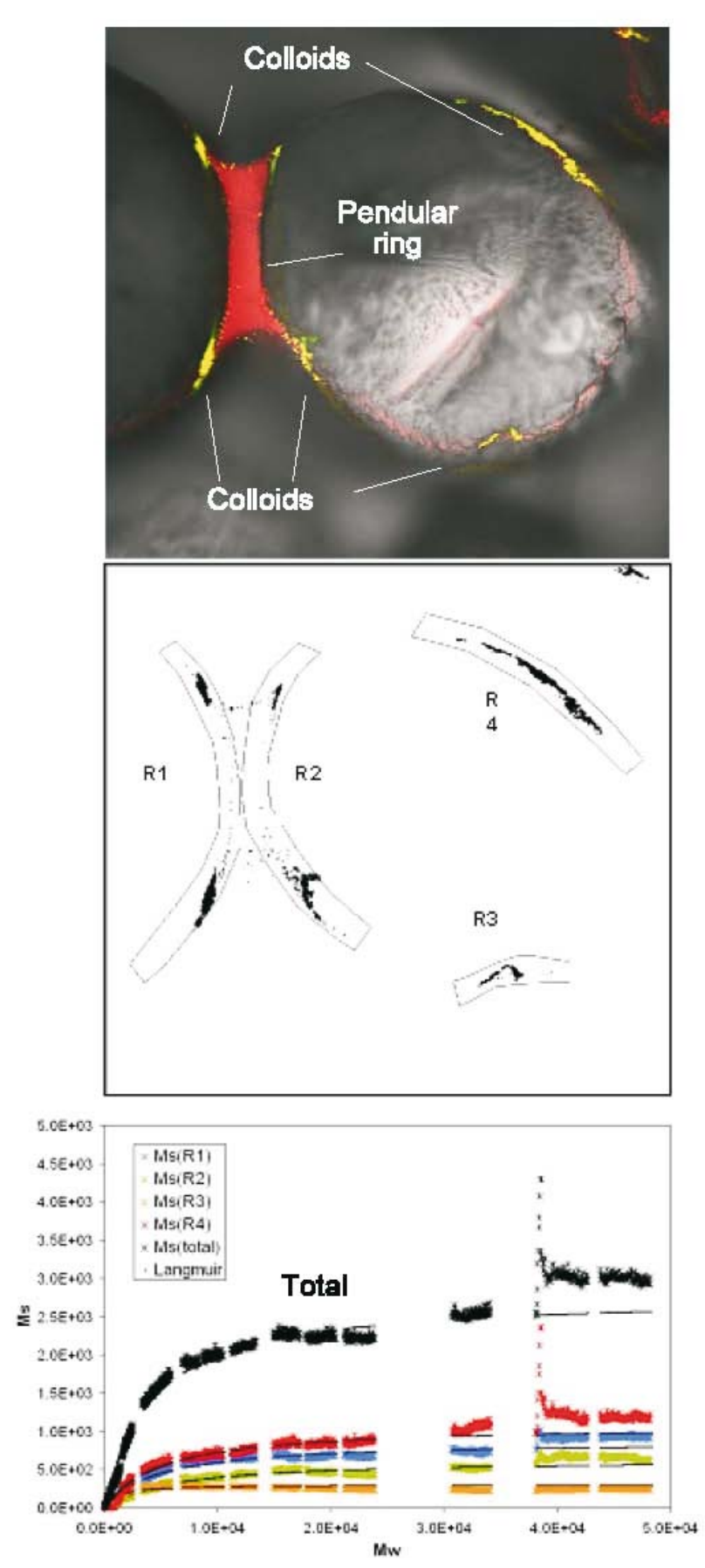

Fig. 5. Fluorescent microsphere colloid attachment at $\mathrm{AW}_{\mathrm{m}} \mathrm{S}$ interface: a) Left: overlay of confocal scanning laser microscope images, with false coloring of colloids (yellow) and dyed water (red) shown on grayscale image of sand grains; b) Right: interfacial regions delineated for particle counting, shown as inverted image (colloids appear black). Image size is $1024 \times 1024$ pixels with a resolution of $0.73 \mathrm{~mm} /$ pixel; c) Langmuir fits (solid lines) of quantification results for the $\mathrm{AW}_{\mathrm{m}} \mathrm{S}$ interfacial regions $\mathrm{R} 1$ through $\mathrm{R} 4$, as well as total of all regions. The perturbation at $\mathrm{Mw}=-37,000$ was due to reloading the input syringe pump.

counted in each image sequence. The area of particles is then calculated as the product of the number of black pixels and the pixel size. Pixels are counted as representing attached particles when colloids are in the same 
region in sequential images; the difference between total and attached particles represents mobile (suspended) particles. For estimating the cumulated mobile particles, velocity and travel distance have been taken into account. Detailed information is given in ZEVI et al. (2006).

The quantification results for the colloidal retention in $\mathrm{AW}_{\mathrm{m}} \mathrm{S}$ interfacial regions $\mathrm{R} 1$ through $\mathrm{R} 4$ in Fig. 4b are shown in Fig. 4c, where the data are fitted to a modified Langmuir form:

$$
\mathrm{M}_{\mathrm{s}}=\frac{\mathrm{KM}_{\mathrm{w}} \mathrm{N}}{1+\mathrm{KM}_{\mathrm{w}}}
$$

where $M_{s}$ is the cumulative amount of colloids attached on the $\mathrm{AW}_{\mathrm{m}} \mathrm{S}$ interface, $\mathrm{M}_{\mathrm{w}}$ is the amount of mobile colloids passing through the image, $\mathrm{N}$ is the total available sites for colloid attachment at the $\mathrm{AW}_{\mathrm{m}} \mathrm{S}$ interface, and $\mathrm{K}$ is the adsorption rate constant. The Langmuir approach assumes maximum retention capacity $(\mathrm{N})$ that corresponds to the available sites on the $\mathrm{AW}_{\mathrm{m}} \mathrm{S}$ interfaces. As can be seen in Fig. 5c, this approach describes colloid attachment well $\left(r^{2}\right.$ in this example is 0.984 for a fit of $\mathrm{M}_{\mathrm{w}}$ up to 24,000). Ongoing work in our group is focused on quantifying the effects of key variables solution chemistry, dynamic flow events, soil properties and colloid/biocolloid surface characteristics - on retention and transport in order to better understand and ultimately ameliorate threats to groundwater posed by contaminant colloids and biocolloids.

\section{References}

Barwick, R.S., Levy, D.A., Craun, G.F., Beach, M.J. \& CALDERON, R.L. 2000. Surveillance for waterborne-disease outbreaks - United States, 1997-1998. CDC Surveillance Summaries 49(ss-4): 1-35.

Crist, J.T., Zevi, Y., McCarthy, J.F., Throop, J.A. \& SteEnhuis, T.S. 2005. Transport and retention mechanisms of colloids in partially saturated porous media. Vadose Zone J. 4: 184-195.

Gao, B., Steenhuis, T.S., Richards, B.K., Zevi, Y., Fehrman, R.L., Dathe, A., Nieber, J.L. \& McCarthy, J.F. 2006. Capillary retention of colloids in unsaturated porous media. (In review)

Macler, B.A. \& Merkle, J.C. 2000. Current knowledge on groundwater microbial pathogens and their control. J. Hydrol. 8(1): 29-40.

MCCarthy, J.F. \& MCKAY, L.D. 2004. Colloid transport in the subsurface: Past, present, and future challenges. Vadose Zone J. 3(2): 326-337.

SUR, J. \& PAK, H.K. 2001. Capillary force on colloidal particles in a freely suspended liquid thin film. Physical Review Letters 86(19): $4326-4329$.

Zevi, Y., Dathe, A., McCarthy, J.F., Richards, B.K. \& Steenhuis, T.S. 2005. Distribution of colloid particles onto interfaces in partially saturated sand. Environ. Sci. Tech. 39(18): 7055-7064.

Zevi, Y., Dathe, A., Richards, B.K. \& Steenhuis, T.S. 2006. Quantifying colloid retention in partially saturated porous media. Water resources research. (In press) 